\title{
An Additional Consideration of Reliability and Validity of the Differentiation of Self Scale in Two Domains
}

\author{
Koji Kudo \\ Department of Educational Psychology, Tokyo Gakugei University, Tokyo, Japan \\ Email: kojikudo@u-gakugei.ac.jp
}

How to cite this paper: Kudo, K. (2018). An Additional Consideration of Reliability and Validity of the Differentiation of Self Scale in Two Domains. Psychology, 9, 2411-2421. https://doi.org/10.4236/psych.2018.910138

Received: July 15, 2018

Accepted: September 14, 2018

Published: September 17, 2018

Copyright $\odot 2018$ by author and Scientific Research Publishing Inc. This work is licensed under the Creative Commons Attribution-NonCommercial International License (CC BY-NC 4.0). http://creativecommons.org/licenses/by-nc/4.0/

\begin{abstract}
This study addressed the insufficient verification of reliability and validity of the Differentiation of Self Scale in Two Domains (DSS-2D). Although the DSS-2D is the first measure in Japan that satisfies the three requirements (corresponding to two domains, applicable to a wide range of adolescents, and a moderate number of items), verification of reliability and validity of the DSS-2D has remained insufficient. Two questionnaire surveys were conducted with university students ( $n=70$ at T1, $n=68$ at T2). The interval of the surveys was one month. The results indicate that the DSS-2D had sufficient test-retest reliability and concurrent validity in each domain of differentiation of self. These results provide support for the DSS-2D as a valid and adequate measure for use in Japan.
\end{abstract}

\section{Keywords}

Differentiation of Self, Domain Specific Validation, Interpersonal Domain, Intrapsychic Domain, Test-Retest Reliability

\section{Introduction}

\subsection{Differentiation of Self Hypothesis}

Differentiation of self, which is one of the main concepts of Bowen's theory, is composed of two domains, differentiation in the interpersonal domain and differentiation in the intrapsychic domain. Interpersonal differentiation is the balance between the desire for individuality and togetherness in interpersonal relationships. Intrapsychic differentiation is the balance between an individual's thoughts and emotions. Those who are less differentiated in the interpersonal domain are likely to have a strong desire for togetherness. Those who are less 
differentiated in the intrapsychic domain are likely to be excessively emotional. According to Bowen, those with low differentiation of self are more vulnerable to stress (Bowen, 1978; Kerr \& Bowen, 1988).

\subsection{Scale Development and Empirical Studies of Differentiation of Self}

This differentiation of self hypothesis has been widely accepted as one of the basic hypotheses in the field of family therapy. However, empirical studies on the differentiation of self have been delayed because of a lack of psychometrically sound measures of differentiation of self (Miller, Anderson, \& Keala, 2004). Bowen described the differentiation of self scale (Bowen, 1978; Kerr \& Bowen, 1988), but this was an attempt to explain various differences in people based on high and low differentiation of self, and rather than being an actual scale, it was a hypothetical one. In the past, many researchers have often used the Personal Authority in the Family System Questionnaire (Bray, Harvey, \& Williamson, 1987) to test Bowen's theory, but it did not exactly measure Bowen's concepts (Miller et al., 2004).

Against this background, two scales have been developed. The first scale is Haber's (1993) Level of Differentiation of Self Scale (LDSS) and the second is Skowron \& Friedlander's (1998) Differentiation of Self Inventory (DSI). These more closely adhere to Bowen's concept of differentiation and their development has enabled the implementation of empirical research on differentiation of self (Miller et al., 2004). Especially, the DSI (Skowron \& Friedlander, 1998) has been used in many studies, and empirical studies on the differentiation of self have been accumulating. The DSI has been improved in terms of its reliability and validity, and it is now the DSI-R (Differentiation of Self Inventory-Revised; Skowron \& Schmitt, 2003). Even now, the concept of differentiation of self has been the focus of a great deal of empirical research (e.g., Knauth, Skowron, \& Escobar, 2006; Lampis, 2016; Peleg \& Yitzhak, 2011; Xue et al., 2018).

\subsection{Current Status of Empirical Studies on the Differentiation of Self Hypothesis in Japan}

To date there have been few empirical studies in Japan on the differentiation of self. Kudo (2018) pointed out the lack of appropriate scales available in Japan as one of the reasons. However, it is difficult to say that it is enough to create a Japanese version of the LDSS or DSI-R, in that the differentiation of self hypothesis assumes the influence of cultural differences. The differentiation of self hypothesis was formed based on Bowen's clinical experience with European Americans in the United States, and many of these subjects had a cultural background that recognized the value of individualism and independence (Gushue \& Constantine, 2003). On the other hand, Japan is considered to be a collectivist culture. It can be said that such cultural differences have some influence on the hypothesis. Therefore, it is considered appropriate to use a scale developed in 
Japan to promote Japan's empirical research on the differentiation of self hypothesis.

According to Kudo (2018), scales for measuring differentiation of self should satisfy the following three requirements. First, it should correspond to the two domains of differentiation. This is one of the fundamental qualities required for the scale because the differentiation of self is a concept consisting of two domains (interpersonal and intrapsychic). Second, the content of the scale items must be applicable to a wide age range encompassed by adolescence. In recent years, the age range of adolescence has been widening because of the earlier start and the later end than before. Within this wide range, unmarried people are also included and so it is better not to include items related to spouses. Additionally, it is desirable to not use difficult terms so that younger adolescents, such as high school students, can understand the item contents. Third, there should not be too many items on the scale. It is important to consider the response burden of survey participants because the scale is likely to be used in conjunction with other measures in many cases. Thus far, in Japan there has not been a scale that satisfies all the three requirements. As a result, Japan's empirical studies on the differentiation of self hypothesis have been lacking.

\subsection{Differentiation of Self Scale in Two Domains and Its Reliability and Validity}

To improve the situation in Japan, Kudo (2018) developed the Differentiation of Self Scale in Two Domains (DSS-2D). The DSS-2D consists of three subscales that correspond to the interpersonal domain (Interpersonal Differentiation Togetherness Scale, Interpersonal Differentiation Individuality Scale, and Adaptive Interpersonal Relationship Scale) and one subscale that corresponds to the intrapsychic domain (Intrapsychic Differentiation Scale). The items were created avoiding the use of difficult-to-understand expressions for younger adolescents, and to make it possible for adolescents of wide age ranges to answer the item. For example, item content such as asking about one's spousal relationship was not included. In total, there are 29 items on the DSS-2D, and this is by no means a large number of items for a scale composed of four subscales. Therefore, it can be said that the DSS-2D is the first scale in Japan that satisfies all three requirements.

However, verification of the reliability and validity of the DSS-2D remains insufficient. For reliability, Kudo (2018) has reported only internal consistency (Cronbach's alpha). The test-retest reliability, which shows DSS-2D's stability over time, has not been evaluated. For validity, Kudo (2018) has reported only a correlation with trait anxiety; that is, only one criterion has been used for validity verification. Although anxiety is a common trait of those with low differentiation of self, it has no direct relation to the concept of each domain of differentiation of self. Since the DSS-2D corresponds to two domains of differentiation, it is preferred to verify the validity corresponding to each domain to examine its va- 
lidity more precisely.

Individuals who are better differentiated in the interpersonal domain are considered to be able to always be themselves without being influenced by their interpersonal relationships. They have their own beliefs and are not easily influenced by others' opinions. Ito \& Kodama (2005) regard the concept of authenticity as living in accordance with one's own opinions and feelings. Harter (2002) considers the ability to share one's thoughts, speak one's mind, and express one's opinions in different relationships as a manifestation of authenticity. Thus, individuals who are better differentiated in the interpersonal domain are considered to be able to maintain a sense of authenticity. Therefore, it can be assumed that there would be a positive correlation between differentiation in the interpersonal domain and sense of authenticity. On the other hand, individuals who are better differentiated in the intrapsychic domain do not react easily and emotionally but are able to think things through even in a stressful situation. They think before deciding. They are able to judge things reasonably. Kagan (1965) states that while there are people who think carefully after gathering more information and cautiously make a conclusion, others will conclude quickly with less information, and such individual differences are seen in the dimension of reflection-impulsivity. From the viewpoint of Kagan (1965), better differentiated individuals in the intrapsychic domain are considered to be reflective and not impulsive. Therefore, it can be assumed that there would be a positive correlation between differentiation in the intrapsychic domain and a reflective tendency. Regarding the validity of the DSS-2D, it is desirable to consider the domain-specific validity based on the above assumptions.

\subsection{The Present Study}

This article addresses the insufficient verification of reliability and validity of the DSS-2D. In doing so, the paper reports its test-retest reliability and domain-specific validity of the DSS-2D. In this study, two questionnaire surveys were conducted to evaluate the test-retest reliability. The domain-specific validity examinations were also carried out in the two surveys. This study contributes to promoting empirical research on the differentiation of self hypothesis in Japan.

\section{Methods}

\subsection{Study Design}

In this study, two questionnaire surveys ( $\mathrm{T} 1$ and $\mathrm{T} 2$ ) with an interval of one month were administered to university students. A survey at $\mathrm{T} 1$ was implemented to examine the validity of the interpersonal domain of the DSS-2D. A survey at T2 was administered to examine the validity of the intrapsychic domain of the DSS-2D. The test-retest reliability of the DSS-2D was evaluated using the results of the two surveys. 


\subsection{Sample Size Calculation}

In the examination of validity, the correlation level was set to $r=0.3$ using Cohen's (1988) effect size criteria (medium size). In the evaluation of the test-retest reliability, the correlation level was set to $r=0.5$ using Cohen's (1988) effect size criteria (large size). In both, the level of significance was set to $p=0.05$ and the power $(1-\beta)$ was set to 0.7 . Calculation of sample size based on these criteria revealed that 67 participants were required for the validity examination and 23 participants were required for the test-retest reliability evaluation.

\subsection{Participants}

Survey participants recruited on a voluntary basis were university students in the Kanto region, who were students of the author. A total of 87 (44 male, 43 female) participated at T1 and 86 (41 male, 45 female) participated at T2. Sixty students ( 32 male, 28 female) participated in both T1 and T2. The participants' ages ranged from 20 to 25 years.

\subsection{Measures}

\subsubsection{Differentiation of Self Scale in Two Domains}

The Differentiation of Self Scale in Two Domains (DSS-2D; Kudo, 2018) is a 29-item self-report scale measuring the degree of differentiation of self in terms of four subscales: Interpersonal Differentiation Togetherness Scale (e.g., I spend a lot of energy trying to get recognized by others), Intrapsychic Differentiation Scale (e.g., I am able to distinguish emotions as emotions and think rationally), Interpersonal Differentiation Individuality Scale (e.g., I have my own beliefs), and Adaptive Interpersonal Relationship Scale (e.g., I am able to enjoy interacting with people). The following internal consistencies have been reported: Interpersonal Differentiation Togetherness Scale (10 items), $\alpha=0.83$; Intrapsychic Differentiation Scale (8 items), $\alpha=0.83$; Interpersonal Differentiation Individuality Scale (6 items), $\alpha=0.79$; and Adaptive Interpersonal Relationship Scale (5 items), $\alpha=0.75$ (Kudo, 2018). Responses are given on a 7-point Likert scale (1 = "does not apply at all" to 7 = "greatly applies"). Higher scale scores indicate a higher degree of differentiation. Note that a higher score on the Interpersonal Differentiation Togetherness Scale indicates a lower desire for togetherness, and people with a lower desire for togetherness are considered to be better differentiated. The DSS-2D was administered in both $\mathrm{T} 1$ and $\mathrm{T} 2$.

\subsubsection{Sense of Authenticity Scale}

The Sense of Authenticity Scale (SAS; Ito \& Kodama, 2005) was used to examine the concurrent validity of the DSS-2D's subscales corresponding to the interpersonal domain (Interpersonal Differentiation Togetherness, Interpersonal Differentiation Individuality, and Adaptive Interpersonal Relationship). As mentioned above, if these subscales are valid in the interpersonal domain, there should be positive correlations between these subscales and the SAS. The SAS is a single scale (7 items). Sufficient reliability and validity have been confirmed for the 
SAS (Ito \& Kodama, 2005). Responses are given on a 5-point Likert scale $(1=$ "does not apply" to 5 = "applies"). Higher scale scores correspond to a more clear sense of authenticity. The SAS was administered at T1.

\subsubsection{Cognitive Reflection-Impulsivity Scale}

The Cognitive Reflection-Impulsivity Scale (CRIS; Takigiku \& Sakamoto, 1991) was used to examine the concurrent validity of the DSS-2D's subscale corresponding to the intrapsychic domain (Intrapsychic Differentiation). If the subscale is valid in the intrapsychic domain, it should have a positive correlation with the CRIS. The CRIS is a single scale (10 items). Sufficient reliability and validity have been confirmed for the CRIS (Takigiku \& Sakamoto, 1991). Responses are given on a 4 -point Likert scale ( $1=$ "does not apply" to $4=$ "applies"). Higher scale scores correspond to being more reflective. The CRIS was administered at $\mathrm{T} 2$.

\subsection{Procedure}

Between May and June 2017, two surveys (T1 and T2) were conducted during lecture periods. The interval of the two surveys was one month. The study was explained both orally and in writing on the coversheet, where it was made clear that their participation was voluntary and they could withdraw from the study at any time. There were no disadvantages to not participating. Personal information on the questionnaire would be protected and their responses to the items would be used only for research purposes. The survey was then conducted.

\subsection{Data Analysis}

First, some item scores were reversed so that higher scores for each DSS-2D subscale would represent a higher degree of differentiation of self. Scale scores were obtained by adding the item scores and then dividing by the number of items. Next, descriptive statistics for all scale scores were calculated, then correlations between the DSS-2D's subscale scores at T1 and T2 were calculated to evaluate test-retest reliability. Next, each of the three DSS-2D subscales corresponding to the interpersonal domain was correlated with the SAS to examine concurrent validity of the DSS-2D for the interpersonal domain. Lastly, the DSS-2D subscale corresponding to the intrapsychic domain was correlated with the CRIS to examine the concurrent validity of the DSS-2D for the intrapsychic domain.

\section{Results}

\subsection{Valid Respondents}

The number of valid respondents for the evaluation of validity was 70 (40 male, 30 female) at T1 and 68 (38 male, 30 female) at T2. The number of valid respondents for the evaluation of test-retest reliability was 56 (29 male, 27 female). Both numbers exceeded the sample size required for the study. The average age was 
20.34 years $(S D=0.78)$ at $\mathrm{T} 1$ and 20.40 years $(S D=0.81)$ at $\mathrm{T} 2$.

\subsection{Descriptive Statistics of Each Scale}

Table 1 shows the mean value, standard deviation, and reliability coefficient (Cronbach's alpha) of each scale. There were no ceiling or floor effects. The internal consistencies (Cronbach's alphas) of these scales were 0.75 or more, showing appropriate values in each scale.

\subsection{Test-Retest Reliability}

Table 2 shows the correlations between the DSS-2D subscales at T1 and the DSS-2D subscales at T2. The correlation coefficient between Interpersonal Differentiation Togetherness at T1 and T2 was $0.86(p<0.001)$. The correlation coefficient between Intrapsychic Differentiation at T1 and T2 was $0.85(p<$ 0.001). The correlation coefficient between Interpersonal Differentiation Individuality at $\mathrm{T} 1$ and $\mathrm{T} 2$ was $0.81(p<0.001)$. The correlation coefficient between Adaptive Interpersonal Relationship at T1 and T2 was $0.79(p<0.001)$. All correlations were significant and positive at the $5 \%$ level. Moreover, all correlations were strong (larger than 0.70 ). The results indicate that the DSS-2D has sufficient test-retest reliability.

\subsection{Domain-Specific Validity}

Table 3 shows the correlations between the scores on the three DSS-2D subscales corresponding to the interpersonal domain and score on the SAS. The correlation coefficient between Interpersonal Differentiation Togetherness and the SAS was $0.38(p=0.001)$. The correlation coefficient between Interpersonal Differentiation Individuality and the SAS was $0.61(p<0.001)$. The correlation coefficient between Adaptive Interpersonal Relationship and the SAS was 0.55

Table 1. Scale means, standard deviations, and Cronbach's alphas.

\begin{tabular}{|c|c|c|c|c|c|}
\hline Scale & Time & $N$ & Mean & $\begin{array}{l}\text { Standard } \\
\text { Deviation }\end{array}$ & $\begin{array}{c}\text { Cronbach's } \\
\text { Alpha }\end{array}$ \\
\hline Interpersonal Differentiation & $\mathrm{T} 1$ & 70 & 3.57 & 0.81 & 0.80 \\
\hline Togetherness & $\mathrm{T} 2$ & 68 & 3.69 & 0.80 & 0.81 \\
\hline \multirow{2}{*}{ Intrapsychic Differentiation } & $\mathrm{T} 1$ & 70 & 4.21 & 0.93 & 0.84 \\
\hline & $\mathrm{T} 2$ & 68 & 4.20 & 0.88 & 0.85 \\
\hline Interpersonal Differentiation & $\mathrm{T} 1$ & 70 & 4.88 & 0.81 & 0.75 \\
\hline Individuality & $\mathrm{T} 2$ & 68 & 4.84 & 0.78 & 0.77 \\
\hline Adaptive Interpersonal & $\mathrm{T} 1$ & 70 & 4.91 & 0.97 & 0.77 \\
\hline Relationship & $\mathrm{T} 2$ & 68 & 4.87 & 1.08 & 0.85 \\
\hline Sense of Authenticity & $\mathrm{T} 1$ & 70 & 3.34 & 0.76 & 0.84 \\
\hline Cognitive Reflection-Impulsivity & $\mathrm{T} 2$ & 68 & 2.70 & 0.59 & 0.86 \\
\hline
\end{tabular}

The scale score is the sum of item scores divided by the number of items. 
Table 2. Correlations between the DSS-2D subscales at T1 and T2.

\begin{tabular}{cc}
\hline & Correlations \\
\hline Interpersonal Differentiation Togetherness & $0.86^{* * *}$ \\
Intrapsychic Differentiation & $0.85^{* * *}$ \\
Interpersonal Differentiation Individuality & $0.81^{* * *}$ \\
Adaptive Interpersonal Relationship & $0.79^{* * *}$
\end{tabular}

$N=56 .{ }^{* *}$ Correlation is significant at $p<0.001$ level.

Table 3. Correlations among the DSS-2D, SAS, and CRIS.

\begin{tabular}{|c|c|c|}
\hline & Sense of Authenticity & $\begin{array}{c}\text { Cognitive } \\
\text { Reflection-Impulsivity }\end{array}$ \\
\hline Interpersonal Differentiation Togetherness ${ }^{a}$ & $0.38^{\star *}$ & \\
\hline Interpersonal Differentiation Individuality ${ }^{a}$ & $0.61^{* * *}$ & \\
\hline Adaptive Interpersonal Relationship ${ }^{a}$ & $0.55^{\star * *}$ & \\
\hline Intrapsychic Differentiation $^{\mathrm{b}}$ & & $0.38^{\star *}$ \\
\hline
\end{tabular}

$(p<0.001)$. All three subscales had a significant positive correlation with the SAS at the 5\% level. Table 3 also shows the correlation between the score on the DSS-2D subscale corresponding to the intrapsychic domain and score on the CRIS. The correlation coefficient was $0.38(p=0.001)$ and showed the subscale had a significant positive correlation with the CRIS at the $5 \%$ level. These results indicate the DSS-2D has sufficient concurrent validity in both the interpersonal and intrapsychic domains.

\section{Discussion and Conclusions}

The aim of this research was to address the insufficient verification of reliability and validity of Kudo's (2018) DSS-2D. For reliability, only internal consistency (Cronbach's alpha) was reported and the test-retest reliability was not. For validity, no examination was made for each domain of differentiation of self. Therefore, this research addressed the verification of the test-retest reliability and the domain-specific validity of DSS-2D. In doing so, two questionnaire surveys were administered to university students with an interval of one month.

First, the test-retest reliability of the DSS-2D was verified. For each subscale of the DSS-2D, significant $(p<0.001)$ positive correlations were found between scale scores at $\mathrm{T} 1$ and those at $\mathrm{T} 2$. All the correlation coefficients exceeded the criterion set initially $(r=0.5)$. The results provide support for the stability of the DSS-2D in terms of test-retest reliability.

Next, the domain-specific validity of the DSS-2D was verified. Scores on the DSS-2D subscales that correspond to the interpersonal domain (i.e., the Interpersonal Differentiation Togetherness, Interpersonal Differentiation Individuality, and Adaptive Interpersonal Relationship scales) showed significant positive 
correlations with the SAS. All the correlation coefficients exceeded the criterion set initially $(r=0.3)$. These results support the validity of the interpersonal domain of DSS-2D. Among these subscales, Interpersonal Differentiation Individuality was most strongly correlated with the SAS $(r=0.61, p<0.001)$. On the other hand, the correlation between Interpersonal Differentiation Togetherness and the SAS was weaker $(r=0.38, p=0.001)$. A more plausible reason for the difference could be that Interpersonal Differentiation Individuality was more directly related to the SAS than Interpersonal Differentiation Togetherness was. Those with a high tendency for individuality are more autonomous and can clearly have a sense of being themselves. On the other hand, those with a low tendency of togetherness do not care excessively about what others think and can have a sense of being themselves. The SAS used in this study measures this sense. As Interpersonal Differentiation Individuality is created based on the high state of individuality, the content of the items could be directly related to the SAS. Therefore, it is considered that the correlation was large. On the other hand, as Interpersonal Differentiation Togetherness is not created based on a low state of togetherness, it is inevitable that the relation with the SAS is somewhat indirect. As a result, it is considered that the correlation was slightly smaller.

Scores on the DSS-2D subscale that corresponds to the intrapsychic domain (Intrapsychic Differentiation Scale) showed a significant positive correlation with the CRIS ( $r=0.38, p=0.001)$. The correlation coefficient exceeded the criterion set initially $(r=0.3)$. This result supports the validity of the intrapsychic domain of the DSS-2D. However, the correlation coefficient was somewhat smaller. Intrapsychic differentiation is the balance between an individual's thoughts and emotions. Individuals who are better differentiated in the intrapsychic domain can think rationally without being emotional even in a stressful situation and are able to judge things calmly. On the other hand, individuals who are less differentiated in the intrapsychic domain tend to be emotional and react impulsively to things. Based on this, the CRIS was used in this study. However, the CRIS contains items related to impulsivity but no items directly corresponding to emotionality. Therefore, the CRIS may have been weakly related to the emotionality aspect of Intrapsychic Differentiation. As a result, it seems that the correlation was slightly weakened.

In conclusion, it can be said that the insufficient verification of reliability and validity of DSS-2D has almost been resolved. The results from this study indicate that the DSS-2D is a valid and reliable measure. It is hoped that the outcome of the present study would be of some use in promoting the accumulation of empirical research on the differentiation of self hypothesis in Japan.

However, this study includes several limitations. First, the survey sample size was small. Although significant results were obtained based on the criteria initially set, it cannot be denied that the sample size was small for the generalization of the results. The DSS-2D is targeted for a wide age range of adolescents, 
but the subjects of this survey were only university students. Second, differentiation of self is related to development (Kerr \& Bowen, 1988), but validity from a developmental point of view has not been studied. Future studies should be conducted with a larger group of adolescents, and validity should be examined using developmental variables such as emotional independence from parents, which is considered to be deeply related to differentiation of self.

\section{Acknowledgements}

This work was supported by JSPS KAKENHI Grant Number JP16K04294.

\section{Conflicts of Interest}

The author declares no conflicts of interest regarding the publication of this paper.

\section{References}

Bowen, M. (1978). Family Therapy in Clinical Practice. Lanham, MD: J. Aronson.

Bray, J. H., Harvey, D. M., \& Williamson, D. S. (1987). Intergenerational Family Relationships: An Evaluation of Theory and Measurement. Psychotherapy: Theory, Research, Practice, Training, 24, 516-528. https://doi.org/10.1037/h0085749

Cohen, J. (1988). Statistical Power Analysis for the Behavioral Sciences (2nd ed.). Hillsdale, NJ: Erlbaum.

Gushue, G. V., \& Constantine, M. G. (2003). Examining Individualism, Collectivism, and Self Differentiation in African American College Women. Journal of Mental Health Counseling, 25, 1-15. https://doi.org/10.17744/mehc.25.1.hagbhguehtb9xkah

Haber, J. (1993). A Construct Validity Study of a Differentiation of Self Scale. Scholarly Inquiry for Nursing Practice, 7, 165-178.

Harter, S. (2002). Authenticity. In C. R. Snyder, \& S. J. Lopez (Eds.), Handbook of Positive Psychology (pp. 382-394). New York: Oxford University Press.

Ito, M., \& Kodama, M. (2005). Sense of Authenticity, Self-Esteem, and Subjective and Psychological Well-Being. Japanese Journal of Educational Psychology, 53, 74-85. https://doi.org/10.5926/jjep1953.53.1_74

Kagan, J. (1965). Impulsive and Reflective Children: Significance of Conceptual Tempo. In J. D. Krumboltz (Ed.), Learning and the Educational Process (pp. 133-161). Chicago: Rand McNally \& Company.

Kerr, M. E., \& Bowen, M. (1988). Family Evaluation: An Approach Based on Bowen Theory. New York: W. W. Norton \& Co.

Knauth, D. G., Skowron, E. A., \& Escobar, M. (2006). Effect of Differentiation of Self on Adolescent Risk Behavior: Test of the Theoretical Model. Nursing Research, 55, 336-345. https://doi.org/10.1097/00006199-200609000-00006

Kudo, K. (2018). A Differentiation of Self Scale in Two Domains for Japan: A Preliminary Study on Its Development and Reliability/Validity. Psychology, 9, 745-759. https://doi.org/10.4236/psych.2018.94047

Lampis, J. (2016). Does Partners' Differentiation of Self Predict Dyadic Adjustment? Journal of Family Therapy, 38, 303-318. https://doi.org/10.1111/1467-6427.12073

Miller, R. B., Anderson, S., \& Keala, D. K. (2004). Is Bowen Theory Valid? A Review of 
Basic Research. Journal of Marital and Family Therapy, 30, 453-466. https://doi.org/10.1111/j.1752-0606.2004.tb01255.x

Peleg, O., \& Yitzhak, M. (2011). Differentiation of Self and Separation Anxiety: Is There a Similarity Between Spouses? Contemporary Family Therapy: An International Journal, 33, 25-36. https://doi.org/10.1007/s10591-010-9137-Z

Skowron, E. A., \& Friedlander, M. L. (1998). The Differentiation of Self Inventory: Development and Initial Validation. Journal of Counseling Psychology, 45, 235-246. https://doi.org/10.1037/0022-0167.45.3.235

Skowron, E. A., \& Schmitt, T. A. (2003). Assessing Interpersonal Fusion: Reliability and Validity of a New DSI Fusion with Others Subscale. Journal of Marital \& Family Ther apy, 29, 209-222. https://doi.org/10.1111/j.1752-0606.2003.tb01201.x

Takigiku, K., \& Sakamoto, A. (1991). Development of a Cognitive Reflection-Impulsivity Scale: Its Reliability and Validity. The 39th Annual Meeting of Japanese Group Dynamics Association. Miyagi, Japan, 39-40.

Xue, Y., Xu, Z., Zaroff, C., Chi, P., Du, H., Ungvari, G. S., Xiang, Y. et al. (2018). Associations of Differentiation of Self and Adult Attachment in Individuals with Anxiety-Related Disorders. Perspectives in Psychiatric Care, 54, 54-63.

https://doi.org/10.1111/ppc. 12200 